\title{
Why Don't You Use A Very Effective Herbal Medicine, Shao-Yao-Gan-Cao-Tang, For Muscle Cramp in Patients on Hemodialysis?
}

\author{
Fumihiko Hinoshita* \\ Department of Nephrology, National Center for Global Health and Medicine, Japan
}

Submission: February 21, 2017; Published: March 02, 2017

*Corresponding author: Fumihiko Hinoshita, Department of Nephrology, National Center for Global Health and Medicine, Japan, Tel: +81 33202

7181; Fax: +81 33202 1038; E-mail: fhinoshi@hosp.ncgm.go.jp

Abbreviations: TCM: Traditional Chinese Medicine; THM: Traditional Herbal Medicine; CAM: Complementary and Alternative Medicine; HD:

Hemodialysis

\section{Introduction}

Traditional Chinese medicine (TCM) or traditional herbal medicine (THM) is generally classified as a kind of complementary and alternative medicine (CAM) in the western world. The majority of THM and their extracted components are seldom regarded as authorized or licensed drugs in USA and in Europe. THM, however, has been used and prescribed for over the centuries in China and other Asian countries. Therefore, herbal medicine-derived drugs have a long and distinguished history, and have been continuously used for centuries to treat various kinds of diseases and/or symptoms. It is natural to find such herbal medicine-derived drugs still in use in modern times because some specific medical drugs or their extracts had originally been taken and made from natural plants all over the world both in East and West. According to a report by Petrucelli RJ II, medicines made from plants have been utilized to treat human ailments from the earliest times throughout the world [1]. Plants contain a vast array of components with biologic activity and about two-thirds of medications released by the US Food and Drug Administration have their origin in plants. Therefore, looking back on the very origin of modern medications, it can be easily imagined and believed that some of the surviving THM that are still in popular use in East Asia are very useful and effective for specific medical problems or symptoms. This appears to be the case in the area of hemodialysis (HD). Here, I describe a typical case of Shao-yao-gan-cao-tang.

A very useful THM, Shao-yao-gan-cao-tang, for muscle cramp

An example of strikingly effective herbal medicine is Shaoyao-gan-cao-tang (Japanese name: Shakuyaku-kanzo-to). This traditional herbal medicine consists of equal amounts of paeony and licorice roots and has a prophylactic anti-cramping effect. Therefore, Shakuyaku-kanzo-to had been used in Japan and China for general muscle painor skeletal muscle tremors since the ancient times. Recently, however, Shakuyaku-kanzo-to has also been well recognized for its specific effectiveness on muscle cramps in hemodialized patients, one of the most common complications of HD and has come to be commonly utilized for more than 20 years since the authors and a few other groups started to make use of it in patients on maintenance HD. In our first report [2], Shakuyaku-kanzo-to (EK-68®, Kracie Pharma, Ltd., Tokyo, Japan) at $6 \mathrm{~g}$ per day was prospectively administered for 4 weeks to five patients on HD who were suffering from frequent muscle cramps. The frequency and severity of cramping before and after the treatment were carefully observed and compared. Skeletal muscle cramps completely disappeared in two of the patients after the start of oral administration of Shakuyaku kanzo-to. Moreover, the frequency of cramping was significantly decreased in two of the remaining three patients after persistent administration. The severity of muscle cramps was also decreased by this treatment in the responsive patients. No serious side effects were detected during the treatment period. The inhibitory effect of Shakuyaku-kanzo-to on muscle contraction was also experimentally examined by using phrenic nerve-diaphragm preparations from male Wistar rats. Differences between the twitch responses were determined when the diaphragms and the nerves were stimulated in the presence and absence of the extract of Shakuyaku-kanzo-to. The results demonstrated that extracts of paeony and licorice roots inhibit contraction of skeletal muscles in rats [2]. A similar and immediate clinical effect of Shakuyaku-kanzo-to for muscular 
cramps during maintenance HD was also reported elsewhere [3]. Though there have been only a few papers on skeletal muscle cramps in patients on maintenance HD in English literatures, Shakuyaku-kanzo-to is now well-known and popular, routinely used for muscle cramps in the patients on HD and other patients with CKD, diabetes mellitus, liver cirrhosis as well as dehydrative aged persons with nocturnal muscle cramps and sports athletes frequently suffering from muscle cramps at least in Japan.

To manage serious muscle cramps during HD, hypertonic saline or glucose may be administered. If muscle cramps frequently occur in every HD session, the sodium concentration of the dialysis solution may be increased to alleviate the cramps. These treatments are certainly effective in most patients during HD sessions, but always helpless whenever HD is not undertaken. There have been a few other suggested treatments with quinine, nifedipine, vitamin E, biotin and other orally administered drugs, which have been reported to prevent cramping in patients undergoing HD [4-13]. Various ingenuous ways of performing HD have also been reported. It looks, however, that these trials did not produce any definite solution to prevent muscle cramps. At least in the respect of significant prophylactic and prompt effect for muscle cramping, no better remedy can be found than oral Shakuyaku-kanzo-to administration. In general, however, few THM have been recognized for their usefulness and clinical significance in Western countries. Most of the physicians dealing with HD who recognize the amazing effect of Shakuyaku-kanzoto in Japan and other Asian countries would feel very sorry for patients on maintenance HD experiencing frequent muscle cramps in Western countries because this excellent medicine has rarely or never been utilized for them.

Fortunately, at least in Japan, many traditional and representative mixtures of extracts from medicinal herbs are made into authorized products by reputable pharmaceutical companies after the herbal mixtures are tested and finally approved by the Ministry of Health, Labor and Welfare. The drug approval process for herbal medicines is almost the same as new Western drugs. There are more than 100 kinds of mixtures of extracts from medicinal herbs that are approved and sold in Japan. Conclusively I believe that more attention should be paid to the effectiveness of such a well-examined THM for patients on HD in the near future and that more clinical studies should be conducted in Western countries as well as in Asian countries.

\section{References}

1. Petrucelli RJ (1994) Monastic incorporation of classical botanic medicines into the Renaissance pharmacopeia. Am J Nephrol 14(4-6): 259-263.

2. Hinoshita F, Ogura Y, Suzuki Y, Hara S, Yamada A, et al. (2003) Effect of orally administered Shao-yao-gan-cao-tang (Shakuyaku-kanzo-to) on muscle cramps in maintenance hemodialysis patients: a preliminary study. Am J Chin Med 31(3): 445-453.

3. Hyodo T, Taira T, Kumakura M, Yamamoto S, Yoshida K, et al. (2002) The immediate effect of Shakuyaku-kanzo-to, traditional Japanese herbal medicine, for muscular cramps during maintenance hemodialysis. Nephron 90(2): 240.

4. Kaji DM, Ackad A, Nottage WG, Stein RM (1976) Prevention of muscle cramps in haemodialysis patients by quinine sulphate. Lancet 2(7976): 66-67.

5. Casciani CU, Caruso E, Cravotto M (1983) Beneficial effects of L-carnitine in post-dialysis syndrome. Curr Ther Res 32: 116-127.

6. Bellinghieri G, Savica V, Mallamace A, Di Stefano C, Consolo F, et al. (1983) Correlation between increased serum and tissue L-carnitine levels and improved muscle symptoms in hemodialyzed patients. Am J Clin Nutr 38(4): 523-531.

7. Peer GM, Blum M, Aviram A (1983) Relief of hemodialysis-induced muscle cramps by nifedipine. Dial Transplant 12: 180-181.

8. Sever MS, Kocak N (1990) Chloroquine phosphate reduces the frequency of muscle cramps during maintenance hemodialysis. Nephron 56(4): 443.

9. Roca AO, Jarjoura D, Blend D, Cugino A, Rutecki GW, et al. (1992) Dialysis leg cramps. Efficacy of quinine versus vitamin E. ASAIO J 38(3): M481-485.

10. Sidhom OA, Odeh YK, Krumlovsky FA, Budris WA, Wang Z, et al. (1994) Low-dose prazosin in patients with muscle cramps during hemodialysis. Clin Pharmacol Ther 56(4): 445-451.

11. Guay DR (2008) Are there alternatives to the use of quinine to treat nocturnal leg cramps? Consult Pharm 23(2): 141-156.

12. El-Hennawy AS, Zaib S (2010) A selected controlled trial of supplementary vitamin $\mathrm{E}$ for treatment of muscle cramps in hemodialysis patients. Am J Ther 17(5): 455-459.

13. Oguma S, Ando I, Hirose T, Totsune K, Sekino H, et al. (2012) Biotin ameliorates muscle cramps of hemodialysis patients: a prospective trial. Tohoku J Exp Med 227(3): 217-223.

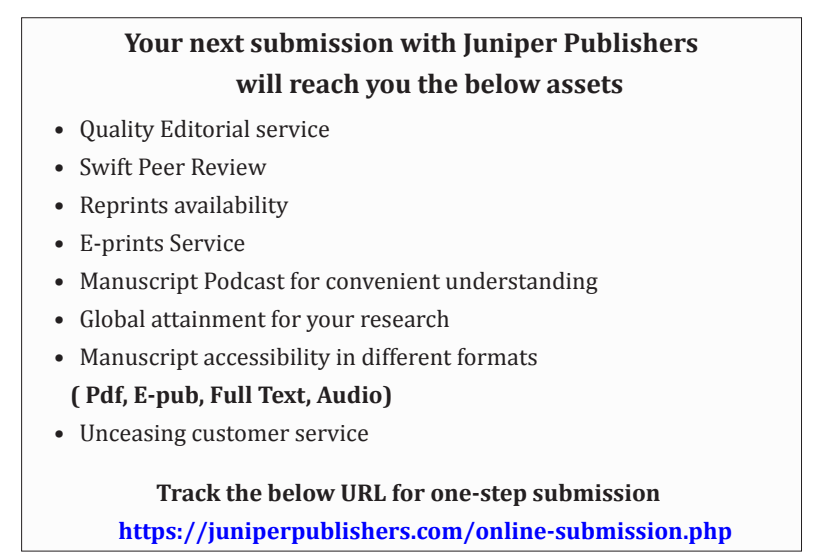

